\title{
POTENCIALIDADES E FRAGILIDADES DO ESTÁGIO CURRICULAR SUPERVISIONADO: CONCEPÇÃO DE DISCENTES E EGRESSOS
}

\author{
POTENTIALITIES AND WEAKNESSES OF THE \\ SUPERVISED CURRICULAR INTERNSHIP: \\ CONCEPTION OF STUDENTS AND GRADUATES
}

\section{POTENCIALIDADES Y FRAGILIDADES DE LA PRÁCTICA CURRICULAR SUPERVISADA: CONCEPCIÓN DE ESTUDIANTES Y GRADUADOS}

\author{
Tierle Kosloski Ramos ${ }^{1}$ \\ Elisabeta Albertina Nietsche ${ }^{2}$ \\ Liege Gonçalves Cassenote ${ }^{3}$ \\ Cléton Salbego ${ }^{4}$ \\ Patrícia Porto Almeida \\ Silvana Bastos Cogo
}

Como citar este artigo: Ramos TK, Nietsche EA, Cassenote LG, Salbego C, Almeida PP, Cogo SB. Potencialidades e fragilidades do Estágio Curricular Supervisionado: concepção de discentes e egressos. Rev baiana enferm. 2019;33:e33076.

Objetivo: identificar potencialidades e fragilidades que emergem no desenvolvimento do Estágio Curricular Supervisionado, na perspectiva de discentes e egressos. Método: estudo qualitativo, descritivo-exploratório com dados coletados de março a maio de 2016 por meio de entrevista semiestruturada. A análise foi de conteúdo do tipo categorial. Resultados: da análise emergiram duas categorias: Potencialidades que emergem no desenvolvimento do Estágio Curricular Supervisionado e Dificuldades enfrentadas durante o Estágio Curricular Supervisionado. Conclusão: o Estágio Curricular Supervisionado promoveu múltiplas influências na formação do enfermeiro, oportunizando experiências atitudinais e relacionais, bem como aprimoramento de conhecimentos teórico-práticos, competências e habilidades; teve como fragilidade a alta demanda de outras disciplinas no mesmo período e dificuldades nas relações interpessoais com os profissionais do serviço e docentes da instituição.

Descritores: Enfermagem. Educação em Enfermagem. Estudantes de Enfermagem. Estágio Clínico.

\footnotetext{
Enfermeira. Mestre em Enfermagem. Professora da Universidade Federal de Santa Maria. Santa Maria, Rio Grande do Sul, Brasil. tierleramos@hotmail.com. https:// orcid.org/0000-0001-7901-3792

2 Enfermeira Obstetra. Doutora em Enfermagem. Professora da Universidade Federal de Santa Maria. Santa Maria, Rio Grande do Sul, Brasil. https://orcid.org/00000002-8006-2038

Enfermeira. Mestre em Enfermagem. Enfermeira Assistencial do Hospital Universitário de Santa Maria. Universidade Federal de Santa Maria. Santa Maria, Rio Grande do Sul, Brasil. https://orcid.org/0000-0003-0833-4016

Enfermeiro. Mestre em Enfermagem. Universidade Federal de Santa Maria. Santa Maria, Rio Grande do Sul, Brasil. https://orcid.org/0000-0003-3734-9970

Estudante de Enfermagem. Universidade Federal de Santa Maria. Santa Maria, Rio Grande do Sul, Brasil.

6 Enfermeira. Doutora em Enfermagem. Professora da Universidade Federal de Santa Maria. Santa Maria, Rio Grande do Sul, Brasil. https://orcid.org/0000-0002-I6868459
} 
Objective: to identify strengths and weaknesses that emerge in the development of the Supervised Curricular Internship, in the perspective of students and graduates. Method: qualitative, descriptive-exploratory study with data collected from March to May 2016 by means of a semi-structured interview. The analysis was of the categorical-type content. Results: the analysis originated two categories: Potentialities that emerge while developing the Supervised Curricular Internship and Difficulties faced during the Supervised Curricular Internship. Conclusion: the Supervised Curricular Internship promoted multiple influences in the nurse's education, allowing for attitudinal and relational experiences, as well as improvement of theoretical and practical knowledge, skills and abilities; the fragility was the high demand from other subjects in the same semester and difficulties in interpersonal relationships with service professionals and faculty of the institution.

Descriptors: Nursing. Education, Nursing. Students, Nursing. Clinical Clerkship.

Objetivo: identificar las potencialidades y fragilidades que surgen en el desarrollo de la Práctica Curricular Supervisada, en la perspectiva de los alumnos y antiguos alumnos. Método: estudio cualitativo, descriptivo-exploratorio con datos recopilados de marzo a mayo de 2016, por medio de entrevista semi-estructurada. El análisis fue de contenido del tipo categórico. Resultados: del análisis surgieron dos categorías: potencialidades que surgen en el desarrollo de la Práctica Curricular Supervisada y dificultades encontradas durante la Práctica Curricular Supervisada. Conclusión: la Práctica Curricular Supervisada promovió múltiples influencias en la formación del enfermero, permitiendo experiencias relacionales y actitudinales, asi como la mejora de los conocimientos teóricos y prácticos, destrezas y habilidades; tuvo como fragilidad, la alta demanda de otras disciplinas en el mismo periodo y las dificultades en las relaciones interpersonales con los profesionales de los servicios y profesores de la institución.

Descriptores: Enfermería. Educación en Enfermería. Estudiantes de Enfermería. Prácticas Clínicas.

\section{Introdução}

As Diretrizes Curriculares Nacionais dos cursos de graduação em enfermagem (DCNEnf) dispõem sobre o perfil do egresso com base nos princípios, fundamentos, condições e procedimentos inerentes à formação do enfermeiro. As DCNEnf abordam competências e habilidades gerais e específicas que devem embasar a formação profissional. Na formação do enfermeiro, existe a obrigatoriedade da inclusão do Estágio Curricular Supervisionado (ECS) nos dois últimos semestres, com 20\% da carga horária total do curso, podendo ser realizado em hospitais, ambulatórios, rede básica de serviços de saúde e comunidades, sob supervisão de um enfermeiro inserido no serviço de saúde e orientação de um docente da instituição de ensino ${ }^{(1)}$.

O desenvolvimento do ECS requer parcerias entre a instituição de ensino e os serviços de saúde. Os cursos de graduação em enfermagem devem prover campos para a realização do ECS. Estes devem viabilizar a inserção dos discentes na realidade de atuação profissional, possibilitando-lhes a concretização da aprendizagem, por meio de atividades teóricas e práticas. Com isso, objetiva-se melhorar a qualificação profissional ${ }^{(2)}$.
Atividades que possibilitem a inserção dos discentes nos cenários de trabalho auxiliam na redução de lacunas entre a teoria e a prática e evidenciam-se enquanto experiências contributivas aos discentes ${ }^{(3)}$. "O ECS insere o indivíduo no cotidiano do trabalho e contribui para a expressão do sujeito profissional" ${ }^{\text {(4:138) }}$. Essa constitui-se etapa fundamental no processo formativo, visto que, além da ressignificação de conhecimentos, há o desenvolvimento do raciocínio crítico, habilidade de comunicação, liderança e tomada de decisões em cenários reais da prática do enfermeiro ${ }^{(5)}$.

Tendo em vista a complexidade que abrange a formação do enfermeiro, ressalta-se a importância de identificar e avaliar as potencialidades e fragilidades do ECS, pois este representa um caminho positivo na construção do perfil do discente-profissional, contribuindo para o desenvolvimento do agir humanista e pensar crítico-reflexivo. O estágio oportuniza aos discentes correlacionar e aplicar sistematicamente saberes teórico-práticos acessados durante o processo formativo, ser capaz de (re)conhecer e intervir frente a situações-problema cotidianas, exercer 
responsabilidade social e compromisso com a cidadania, como promotores da saúde integral do ser humano ${ }^{(1)}$. Em tempos de discussões e reformulação para novas DCNEnf, torna-se válido entender, junto aos discentes e egressos, as repercussões do ECS no processo formativo. Entende-se que este pensar torna possível as futuras reformulações de currículos, visando formar profissionais cada vez mais qualificados para inserção no mercado de trabalho, além de representar com magnitude a disciplina enfermagem. Em vista disso, este estudo questiona: Quais as potencialidades e fragilidades que emergem durante o desenvolvimento do Estágio Curricular Supervisionado no processo de formação dos discentes e egressos de um curso de graduação em enfermagem?

O objetivo deste artigo é identificar as potencialidades e fragilidades que emergem no desenvolvimento do Estágio Curricular Supervisionado na perspectiva de discentes e egressos.

\section{Método}

Estudo descritivo e exploratório, com abordagem qualitativa, desenvolvido em um curso de graduação em enfermagem de uma Universidade Federal do Estado do Rio Grande do Sul, Brasil, no período de março a maio de 2016.

O curso já atuou com sete organizações curriculares. A última passou a vigorar no primeiro semestre de 2016, não tendo ainda desenvolvido o ECS. O ECS passou a fazer parte do curso no quarto currículo e está vigente desde 1998. O sexto currículo, com duração de oito semestres, vigente no momento da pesquisa, contava com duas disciplinas de ECS. A primeira, no sétimo semestre, com carga horária total de 210 horas, e a segunda, no oitavo semestre, com carga horária total de 600 horas. Isso, concomitantemente a duas disciplinas de Trabalho de Conclusão de Curso (TCC): a primeira, no sétimo semestre, com carga horária total de 60 horas, e a segunda, no oitavo, com carga horária total de 60 horas.

Participaram da pesquisa discentes do curso de enfermagem e egressos cursando residência. A seleção ocorreu mediante os seguintes critérios de inclusão: os discentes deveriam estar regularmente matriculados no primeiro semestre de 2016, na disciplina Estágio Supervisionado II (ECS II), equivalente ao $8^{o}$ e último semestre do curso; os egressos deveriam estar cursando o Programa de Residência Multiprofissional na mesma instituição no momento da coleta de dados. Foram excluídos da pesquisa: discentes do curso de graduação que faziam parte do grupo de pesquisa em que o projeto foi apresentado e discutido, por terem tido acesso privilegiado ao processo de construção da pesquisa; e os discentes que participaram do teste piloto do instrumento de coleta de dados.

No momento da coleta de dados, estavam matriculados 17 discentes na disciplina ECS II. Deste quantitativo, 3 não manifestaram interesse em participar do estudo, 2 foram excluídos por terem participado do teste piloto e 2, por fazerem parte do grupo de pesquisa. Assim, a amostra foi constituída de 10 discentes. Quanto aos egressos, 17 estavam cursando residência. Destes, 9 não manifestaram interesse em participar do estudo. A amostra foi composta de 8 enfermeiros egressos. Desse modo, o estudo totalizou 18 participantes.

A coleta de dados ocorreu por meio de entrevista semiestruturada. Estas foram agendadas em data, hora e local de acordo com a disponibilidade dos participantes. O roteiro de entrevista foi composto por 5 questões sociodemográficas e 12 questões sobre ECS. Foi realizado teste piloto com 3 participantes: 2 discentes e 1 egresso. As entrevistas foram gravadas e transcritas na íntegra. Os participantes foram identificados pela letra "D" (discente) e "R" (residente), seguidas de número indicativo da sequência da realização das entrevistas (D1, D2,..R1, R2).

Foi utilizada a técnica de análise de conteúdo do tipo categorial $^{(6)}$. Na etapa de pré-análise foi realizada a leitura flutuante das entrevistas transcritas, visando identificar os núcleos de sentido. Concomitantemente se iniciou o processo de destaque (marcação) desses núcleos que respondiam ao objeto de estudo. Na etapa de exploração do material, foi realizada leitura aprofundada, procedendo-se à identificação e 
extração das unidades de registro e de contexto. Teve início o processo de agrupamento por semelhança, atendendo aos critérios de exaustividade, representatividade, homogeneidade, pertinência e exclusividade. Na etapa de tratamento e interpretação, realizou-se o agrupamento das unidades, formando-se as categorias analíticas.

A pesquisa atendeu aos preceitos da Resolução n. 466/2012, que norteia pesquisas com seres humanos, e obteve aprovação do Comitê de Ética em Pesquisa (CEP), da instituição de filiação do estudo, conforme Parecer n. 1.370.240, Certificado de Apresentação para Apreciação Ética n. 51624315.5.0000.5346.

\section{Resultados}

Após a análise das entrevistas, emergiram duas categorias, que se encontram descritas na sequência: Potencialidades que emergem no desenvolvimento do ECS e Dificuldades enfrentadas durante o Estágio Curricular Supervisionado.

\section{Potencialidades que emergem no desenvolvimento do ECS}

A atuação dos discentes nos serviços de saúde encontra-se diretamente relacionada ao desenvolvimento de habilidades e competências ao longo do ECS. Essa etapa formativa possibilita o aprimoramento e a consolidação de conhecimentos teórico-práticos. O ECS favorece o amadurecimento do discente diante da realidade profissional que se aproxima, possibilitando o fortalecimento da responsabilidade e a autonomia:

\footnotetext{
Eu acho que o estágio veio para aprimorar o meu conbecimento. (D1).

Contribui na autonomia, de ter mais poder de decisão $e$ da responsabilidade também; seria um teste como se fosse eu a enfermeira. (R4).

Autonomia. A gente tem que ter iniciativa perante as situações. Tem muitas coisas que o enfermeiro tem que ter, mas esta é uma das principais. (D2).

Estou me sentindo mais responsável na questão da saúde do paciente, mais atento a erros, a negligência. (D9).
}

A imersão na realidade do trabalho do enfermeiro possibilita que os discentes estabeleçam relações interpessoais intrínsecas ao ambiente em que estão inseridos. Entre elas, revela-se a oportunidade de interagir com discentes de outras instituições, com as equipes, enfermeiras supervisoras, docentes orientadoras e usuários dos serviços:

\begin{abstract}
Foi muito bom! Eu acho que me acrescentou muito como profissional o jeito das pessoas agirem como profissionais. Eu me inspirei nelas, pela maneira como tratavam os pacientes. (R6).

Se acontecia algo novo, ela [enfermeira supervisora] me mudava de paciente. Foram diversos casos que tu tens que desempenhar cuidados diferentes [...] Estudava junto [a enfermeira supervisora]. Não era como: "Eu sou um ser maior que você". (R1).
\end{abstract}

\begin{abstract}
A professora sempre foi aberta ao que eu trazia. Ela explicava, conversava, a gente trabalhava junto. Ela via minha posição sobre coisas que aconteciam [...] então acho que eu tive uma boa experiência [...] teve diálogo, sempre foi aberta. (R2).
\end{abstract}

Eu sempre tento tratar de uma forma boa e nunca tive problema com usuário, pelo contrário. (D7).

O fato de interagir com acadêmicos de outras instituições é muito bom. Permite compartilhar ensinamentos, conbecimentos. (D4).

A vivência diária da rotina dos serviços de saúde, em especial do enfermeiro, possibilita ao discente observar, compreender e contribuir com as atividades desenvolvidas nos locais em que se encontra inserido. Assim, o ECS é um facilitador para a tomada de decisões nos locais de atuação profissional, bem como para o autoconhecimento, autocorreção de comportamentos, além do preparo para o mercado de trabalho e o entendimento do funcionamento do Sistema Único de Saúde (SUS):

Porque a gente procura sempre, no decorrer do estágio, corrigir alguma coisa que não esteja indo bem. (D3).

Com questões de conbecer-me como futuro profissional, como pessoa, o caráter, a responsabilidade. (D6).

Eu consigo ver também pra onde eu quero seguir, por fazer parte desses estágios nesses campos, o que eu não me vejo fazendo no futuro. (D5).

Ver o SUS como, foi ver o SUS na sua totalidade mesmo, $e$ ver que o programa funciona, mas é demorado. Então fo $i$ um novo olhar que eu tive sobre o SUS. (D9). 


\section{Dificuldades enfrentadas durante o Estágio Curricular Supervisionado}

Por meio da aproximação com o mundo do trabalho, o discente coloca-se como enfermeiro e identifica fragilidades que necessitam atenção. Por vezes, as fragilidades envolvem conhecimentos (âmbito teórico) como procedimentos (âmbito prático), visto que essa etapa propicia o enfrentamento de situações ainda não vivenciadas. As fragilidades identificadas incluem a necessidade de aprofundar conhecimentos, dificuldades na realização de procedimentos e a alta carga horária:

Precisei buscar um conbecimento maior, foi algo mais específico, dai eu precisei me aprofundar mais naquilo, eu peguei o livro na biblioteca. (D1).

Tu fazes [procedimentos] de um jeito, porque tu aprendeste daquele jeito. Então vem outro e fala: "Não, está tudo errado, faz de outro jeito". Desde que tu faças a técnica correta de formas diferentes, está tudo bem. (R1).

Porque tu tens que se matar estudando, com toda aquela carga borária que tem que fazer nos últimos anos. Eu acho que uma vulnerabilidade é a carga borária [alta do estágio]. (R1).

As relações interpessoais possibilitam o aprimoramento dos discentes durante essa etapa. Quando não ocorrem de forma positiva, podem comprometer o desenvolvimento e a qualidade do ECS. Nesse contexto, emergem dificuldades de convivência e comunicação, decorrentes das relações com o enfermeiro supervisor, com a equipe de saúde, a docente orientadora e os usuários dos serviços: A minha dificuldade foi o acesso ao profissional enfer-
meiro [...] porque eu, tendo dificuldade de acessar ao
profissional enfermeiro, ficarei carregada de dúvidas
que vou buscar sozinha. E essas dúvidas talvez não me
completem e não sejam sanadas. (D2).

Eu tive problema com um funcionário. Às vezes, eles não te enxergam como enfermeiro ali. (D3).

As equipes são muito fechadas. Eu acho que é isso que causa um pouco de medo. Tinham técnicos que nem se comunicavam. Tratavam-me como empecilho. (R1).

A minha [docente] orientadora em relação ao estágio, eu não tive muito contato, sempre tentei trazer essas questões do problema de lá, nunca tive uma supervisão dela nos estágios. E também ela não demonstra muito interesse em saber. Não tenho muito diálogo com ela em relação ao estágio. (D7).
Com os usuários também, alguns eram um pouco "grossos". Às vezes, eles chegavam e diziam: "Eu quero consulta". (D1).

\section{Discussão}

As competências e habilidades na formação em enfermagem fazem parte de um conjunto que visa preparar o futuro enfermeiro para enfrentar as transformações do mundo do trabalho ${ }^{(7)}$. Assim, por meio do desenvolvimento de atividades gerenciais, educativas e assistenciais durante o ECS, o discente passa a desenvolver autonomia, bem como tomada de decisão, conforme se observa entre as potencialidades relatadas tanto por egressos como por discentes. O processo de autonomia ocorre gradualmente, de acordo com a singularidade do discente ${ }^{(8)}$. Tanto a autonomia como as habilidades e competências são produtos essenciais no processo formativo, uma vez que a profissão exige uma assistência pautada na qualidade e resolutividade em saúde para o indivíduo, a família e a coletividade. Para isso, é indispensável que o discente compreenda a corresponsabilidade com sua formação, conforme exposto no relato do participante D2.

O Código de Ética dos Profissionais de Enfermagem descreve que o profissional de Enfermagem atua com autonomia e em consonância com os preceitos éticos, legais, técnico-científicos e teórico-filosóficos ${ }^{(9)}$. Dessa forma, a inserção nos cenários de prática durante o ECS resulta em uma estratégia de ensino-aprendizagem significativa, por meio da qual se articula a teoria e a prática, motivando os discentes e despertando suas potencialidades ${ }^{(10)}$. Esse período possibilita-lhes a busca por fundamentação científica para sustentar suas ações, evidenciando uma reaproximação com atividades teórico-práticas vivenciadas em outros momentos da formação, bem como a busca por novos aprendizados.

A identidade profissional é construída durante a relação do indivíduo com o seu eu, com os outros e com o espaço de trabalho em que está inserido. Assim, o cenário em que o discente encontra-se durante o ECS, pode influenciar em 
suas atitudes, transformando e expressando sua afirmação profissional $^{(4)}$. Dessa forma, além da corresponsabilização do discente, as contribuições do docente orientador e do enfermeiro supervisor são significativas para promover reflexões no discente acerca de sua identidade profissional. Além disso, possibilita-lhe o autoconhecimento e a autocorreção enquanto futuro enfermeiro, por meio da identificação das suas potencialidades e fragilidades. A presença de profissionais e da docente durante o ECS, conforme se observa no relato dos participantes R6, R1 e R2, são decisivas para a aprendizagem. Também, ao vivenciar o trabalho do enfermeiro, o discente reflete acerca dos campos de atuação que lhe interessam dar continuidade, por meio do ensino formal, se na assistência ou na gerência.

As mudanças paradigmáticas, as transformações e as conquistas necessárias para que os princípios do SUS sejam respeitados influenciaram na (re)construção dos projetos pedagógicos dos cursos, que devem estar em consonância com a reforma sanitária e as políticas de saúde ${ }^{(11)}$. Desse modo, entender o funcionamento do SUS é essencial e vem ao encontro dos objetivos das DCNEnf, que apontam que "A formação do enfermeiro deve atender as necessidades sociais de saúde, com ênfase no Sistema Único de Saúde (SUS) e assegurar a integridade da atenção e a qualidade e humanização do atendimento"(1:3). A teoria adquirida em sala de aula consolida-se quando o discente encontra-se imerso no sistema, o que oportuniza o entendimento do seu funcionamento com plenitude, identificando seus fatores potenciais e limitantes.

Em um estudo desenvolvido com enfermeiros supervisores, identificou-se que os discentes apresentam embasamento teórico limitado $^{(12)}$. Em consonância, observa-se que as dificuldades de cunho teórico-prático, referidas pelos participantes D1 e R1, são percebidas enquanto vulnerabilidades, uma vez que o discente espera chegar ao ECS dominando os conteúdos e procedimentos apreendidos e/ou realizados ao longo da formação. Entretanto, a aquisição e/ou o aprofundamento da fundamentação científica caracteriza-se como corresponsabilização do discente com sua formação. As DCNEnf corroboram o exposto ao apontarem, entre as competências e habilidades gerais à educação permanente, a capacidade de que o profissional aprenda continuamente, tanto na formação quanto na prática ${ }^{(1)}$. A alta carga horária durante o ECS, referida pelos participantes como dificuldade, vem acompanhada da realização de outras disciplinas e atividades extracurriculares. Assim, quando o discente realiza uma gama de atividades, não consegue aprofundar seus conhecimentos e se frustra por não estar desempenhando tal atividade com plenitude.

A dificuldade de realizar procedimentos práticos também foi referida pelos participantes. Destaca-se que, ao longo do processo formativo, muitas vezes, as turmas de aulas práticas são compostos por um número elevado de alunos e nem todos têm a oportunidade de realizar/ observar todos os procedimentos, particularmente aqueles privativos do enfermeiro. Os discentes são negativamente afetados pelo excesso de alunos, apontando-se a necessidade de se estabelecer estratégias diante de situações que envolvam grandes grupos na prática clínica ${ }^{(3)}$. Entretanto, o ECS permite o entrelaçamento da teoria e da prática que serão determinantes para a formação, qualificação e elaboração do perfil do futuro enfermeiro ${ }^{(12)}$. O ECS possibilita ao discente o aperfeiçoamento da prática, visto que ele realiza e aperfeiçoa os procedimentos desenvolvidos e assimilados em momentos anteriores durante a formação ${ }^{(4)}$.

O trabalho do enfermeiro é amplo e envolve múltiplas dimensões em uma rede de relações e interações na qual o ser humano está inserido ${ }^{(13)}$. Nesse contexto, ao vivenciar o ECS, o discente necessita estabelecer diferentes relações interpessoais para desenvolver suas atividades. No entanto, as relações que permeiam essa etapa dependem de diversos fatores, incluindo o cenário e a equipe de saúde e, principalmente, a inserção e adaptação do discente nesse meio. Assim, é essencial que todos os envolvidos estejam engajados coletivamente, compartilhando conhecimentos e experiências. Em contraponto, limitações nas relações interpessoais presentes 
no ECS podem resultar em malefícios ao serviço/ usuário e à formação do discente.

$\mathrm{Na}$ experiência do ECS, a oportunidade de trabalhar entre discentes é evidenciada como fator potencializador para o processo de aprendizagem $^{(14)}$. O contato com discentes de outras instituições foi mencionado pelos participantes como fator positivo durante o ECS. Essas relações constroem o ambiente produtivo da realidade profissional, no qual o discente fica imerso, sem distinção e rotulações, assemelhando-se às futuras relações com colegas de trabalho no âmbito profissional.

Sobre o ECS, um estudo argumenta: "Por se tratar de uma disciplina que exige a interação das instituições de saúde e ensino, o ECS sofre influência direta de ambas as partes, tanto de forma negativa como positivamente",(12:49). Quanto ao relacionamento do discente com a equipe profissional, com o enfermeiro supervisor e o docente orientador, observou-se, nos relatos dos participantes, que houve experiências tanto negativas quanto positivas. Conforme referido por alguns participantes, o "exemplo de outros profissionais" reflete na formação do discente. Assim, por meio da convivência com os profissionais atuantes nos serviços, o discente passa a ter uma visão crítica e reflexiva acerca do profissional que está moldando.

Em estudo realizado na perspectiva de discentes, egressos e docentes, identificou-se que o enfermeiro supervisor desempenha papel fundamental no processo de ensino-aprendizagem durante o ECS. Também se constatou que a ausência do docente é um fator que dificulta a relação entre discentes e egressos. Dessa forma, aponta-se a necessidade de articulação entre o enfermeiro (representante da instituição de estágio) e o docente (figura relacionada à instituição de ensino), com o objetivo de ter ressonância entre esses dois profissionais e para proporcionar melhor aprendizado ao discente ${ }^{(15)}$.

A equipe muitas vezes não está preparada para receber discentes na rotina de serviço em que se encontra. Por maiores que sejam os esforços que o discente desenvolva para gerar um bom relacionamento, não são vistos com "bons olhos" e, muitas vezes, são rotulados como "estagiários". No que diz respeito às relações com os usuários dos serviços, que se encontram muitas vezes em situações de adoecimento/vulnerabilidade, nem sempre têm cunho positivo ou resultam em uma boa experiência. Assim, mesmo essas situações sendo frustrantes, enfatiza-se a importância de vivenciá-las no decorrer da graduação. Elas possibilitam ao discente a oportunidade de refletir e posicionar-se em relação às condutas que devem ser adotadas nessas situações.

Outro estudo enfatiza: "Sobre a importante integração entre a academia, os serviços de saúde e a comunidade, é fundamental discuti-la constantemente para a formação profissional nos diversos níveis de atenção." ${ }^{(16: 330)}$ Para que docentes, serviço e estudantes participem ativamente do processo de formação, é indispensável que o ECS seja conduzido por esses sujeitos de modo adequado ${ }^{(17)}$. Nesse contexto, enfatiza-se a importância de o enfermeiro supervisor, bem como do docente orientador, atuarem como intermediadores dessas situações. A ausência de interesse, bem como as dificuldades de relação pela falta de abertura ou, ainda, divergência de pensamentos encontram-se presentes nas relações entre enfermeiro supervisor, docente orientador e discentes.

Estudo aborda que "A educação de qualidade está relacionada à ampliação das interações e associações sistêmicas e à capacidade de fortalecer a interlocução com a realidade complexa e em constante mudança” ${ }^{(18: 6)}$. Isto posto, destaca-se a relevância de que todos os envolvidos na realização do ECS - discentes, serviço, gestores, instituições de ensino - encontrem-se em constante integração e sejam comprometidos com a formação. Ainda existem muitos desafios a serem superados, porém, somente por meio da identificação de fragilidades, os sujeitos envolvidos podem empenhar-se na melhoria do processo de realização do ECS.

Salienta-se que o presente estudo possui, como limitação, o fato de ser realizado apenas na perspectiva dos discentes e egressos participantes do ECS, não contemplando todos os 
sujeitos envolvidos no desenvolvimento dessa etapa, entre eles enfermeiros supervisores, docentes orientadores e gestores. É importante, para o aprofundamento do conhecimento, que outros estudos contemplem todos os sujeitos envolvidos no desenvolvimento do ECS.

\section{Conclusão}

O estudo possibilitou identificar, na perspectiva de discentes e egressos, potencialidades e dificuldades vivenciadas durante o desenvolvimento do ECS. Entre as potencialidades, identificou-se que o ECS apresenta-se como o período em que os discentes podem desenvolver habilidades e competências, incluindo o desenvolvimento de autonomia, a tomada de decisão e o aprimoramento de conhecimentos teórico-práticos. Assim, a presença do docente e do enfermeiro possibilitam-lhes a autorreflexão acerca do seu perfil profissional, bem como a autocorreção. Entre as dificuldades evidenciadas, foram mencionadas pelos participantes as limitações teórico práticas, a alta demanda de carga horária de outras disciplinas cursadas no mesmo período de desenvolvimento do ECS, bem como as relações interpessoais com os profissionais do serviço e docentes da instituição, durante a imersão nos serviços de saúde. Assim, em tempos de novas diretrizes curriculares, a identificação das potencialidades e fragilidades, não só acerca do ECS, mas também do processo formativo em sua totalidade, possibilita que as instituições repensem e organizem seus projetos político-pedagógicos pautados na qualificação da formação e consequentemente da assistência prestada por esses futuros profissionais.

Entre os benefícios deste estudo, evidencia-se a identificação de possíveis potencialidades e fragilidades que os discentes vivenciam durante o ECS. Esse reconhecimento deve oportunizar (re)pensar estratégias para o preenchimento de possíveis lacunas na formação discente, bem como promover o fortalecimento das potencialidades que permeiam o ECS.

\section{Colaborações:}

1 - concepção, projeto, análise e interpretação dos dados: Tierle Kosloski Ramos, Elisabeta Albertina Nietsche e Liege Gonçalves Cassenote; 2 - redação do artigo e revisão crítica relevante do conteúdo intelectual: Tierle Kosloski Ramos, Elisabeta Albertina Nietsche, Cléton Salbego e Patrícia Porto Almeida;

3 - aprovação final da versão a ser publicada: Tierle Kosloski Ramos, Elisabeta Albertina Nietsche e Silvana Bastos Cogo.

\section{Referências}

1. Brasil. Resolução n. 3, de 7 de novembro de 2001. Institui as Diretrizes Curriculares Nacionais do Curso de Graduação em Enfermagem [Internet]. Brasília, (DF); 2001 [cited 2017 nov 10]. Available from: http://portal.mec.gov.br/cne/arquivos/pdf/ CES03.pdf

2. Baldoino AS, Veras RM. Analysis of Servicelearning activities adopted in health courses of Federal University of Bahia. Rev Esc Enferm USP [Internet]. 2016 [cited 2017 Nov 17];50(spe):17-24. Available from: http://www.scielo.br/pdf/reeusp/ v50nspe/0080-6234-reeusp-50-esp-0017.pdf

3. Arkan B, Ordinb Y, Yilmazc D. Undergraduate nursing students' experience related to their clinical learning environment and factors affecting to their clinical learning process. Nurse Educ Pract. 2018;29:127-32. DOI: 10.1016/j.nepr.2017.12.005

4. Lüdke M, Almeida EESA, Silva ALB. Contribuciones de la Etapa Supervisada para la Formación de la Identidad Profesional de las enfermeras. Cultura de los cuidados. 2017;21(48):131-9. DOI: 10.14198/ cuid.2017.48.15

5. Esteves LSF, Cunha ICKO, Bohomol E, Negri EC. Supervised internship in undergraduate education in nursing: integrative review. Rev Bras Enferm. 2018;71(Suppl 4):1740-50. DOI: 10.1590/0034-7167-2017-0340

6. Bardin L. Análise de conteúdo. São Paulo: Edições 70; 2016.

7. Vieira MA, Souto LES, Souza SM, Lima CA, Ohara CVS, Domenico EBL. Diretrizes Curriculares Nacionais para a área da enfermagem: o papel das competências na formação do enfermeiro. 
Rev Norte Mineira Enferm [Internet]. 2016 [cited 2017 Dec 16];5(1):105-21. Available from: http://www.renome.unimontes.br/index. $\mathrm{php} / \mathrm{renome} / \mathrm{article} / \mathrm{view} / 102 / 148$

8. Alvarez LNR, Moya JLM. The student-nurse pedagogical relationship: a hermeneuticphenomenological study. Texto Contexto Enferm. 2017;26(2):e00560016. DOI: 10.1590/010407072017000560016

9. Conselho Federal de Enfermagem. Resolução n. 564/2017, de 6 de novembro de 2017. Aprova o novo Código de Ética dos Profissionais de Enfermagem [Internet]. Brasília (DF); (2017) [cited 2017 Dec 16]. Available from: http://www.cofen. gov.br/resolucao-cofen-no-5642017_59145.html

10. Peres CRFB, Marin MJS, Tonhom SFR, Marques MLSF. Current challenges in nursing education: the professor's perspective. Rev Rene. 2018;19:e3160. DOI: $10.15253 / 2175-6783.2018193160$

11. Winters JRF, Do Prado ML, Heidemann ITSB. Nursing education oriented to the principles of the Unified Health System: perception of graduates. Esc Anna Nery. 2016;20(2):248-53. DOI: $10.5935 / 1414-8145.20160033$

12. Souza DJ, Faria MF, Cardoso RJ, Contim D. Supervised intership under the nurses' optical supervisory. Rev Enferm Atenção Saúde. 2017 jan-jun;6(1):39-51. DOI: 10.18554/reas.v6i1.1677

13. Borges AMM, Brito RS, Chagas SNF. Percepção dos estudantes de graduação em enfermagem sobre o trabalho do enfermeiro. R Enferm Cent O Min. 2016;6(3):2421-9. DOI: 10.19175/recom.v6i3.1118
14. Jansson I, Ene KW. Nursing students' evaluation of quality indicators during learning in clinical practice. Nurse Educ Pract. 2016 Sep;20:17-22. DOI: 10.1016/j.nepr.2016.06.002

15. Rigobello JL, Bernardes A, Moura AA, Zanetti ACB, Spiri WC, Gabriel CS. Estágio Curricular Supervisionado e o desenvolvimento das competências gerenciais: a visão de egressos, graduandos e docentes. Esc Anna Nery. 2018;22(2):e20170298. DOI: 10.1590/2177-9465-EAN2017-0298

16. Garcia SD, Vannuchi MTO, Garanhani ML, Sordi MRL. Internato de enfermagem: conquistas e desafios na formação do enfermeiro. Trab Educ Saúde. 2018;16(1):319-36. DOI: 10.1590/1981-7746-sol00105

17. Marchioro D, Ceratto PC, Bitencourt JVOV, Martini JG, Silva Filho CC, Silva TG. Estágio Curricular Supervisionado: relato dos desafios encontrados pelos(as) estudantes. Arq Ciênc Saúde UNIPAR. 2017 May-Aug;21(2):119-22. DOI: 10.25110/ arqsaude.v21i2.2017.5912

18. Backes DS, Zamberlan C, Siqueira $\mathrm{HCH}$, Backes MTS, Sousa FGM, Lomba MLLF. Quality nursing education: a complex and multidimensional phenomenon. Texto Contexto Enferm. 2018;27(3):e4580016. DOI: 10.1590/0104070720180004580016

Recebido: 16 de agosto de 2019

Aprovado: 21 de outubro de 2019

Publicado: 16 de março de 2020

A Revista Baiana de Enfermagem utiliza a Licença Creative Commons - Atribuição-NãoComercial 4.0 Internacional. https://creativecommons.org/licenses/by-nc/4.0/

Este artigo é de acesso aberto distribuído sob os termos da Licença Creative Commons (CC BY-NC). Esta licença permite que outros remixem, adaptem e criem a partir do seu trabalho para fins não comerciais. Embora os novos trabalhos tenham de lhe atribuir o devido crédito e não possam ser usados para fins comerciais, os usuários não têm de licenciar esses trabalhos derivados sob os mesmos termos. 